\title{
Reliability Analysis of the Railway Time Synchronization Network Based on Bayesian
}

\author{
Youpeng Zhang, Feng Wang, Shan Zhang and Li Lan \\ School of Automation and Electrical Engineering, \\ Lanzhou Jiaotong University, Lanzhou 730070, China \\ 506541066@qq.com
}

\begin{abstract}
With the high speed railway speed increasing and driving distance shortening in China, the time conformity of subsystems of railway is becoming more significant to railway traffic safety. The railway time synchronization network (RTSN), the medium carrying and broadcasting time information, is responsible to convey the information of internal clock of railway and its function poses direct influence on the time conformity of subsystems of railway. This paper firstly analyzes the RTSN, including its functions, the differentiation of layers in system, and the hardware framework. Then combining factors like equipment redundancy and common cause failure, etc, the paper collects a large number of data by gathering field data and experts suggestions and Bayesian (BN) is established. Thus reliability analyses of RTSN is conducted and, by inference, its weak links can be discovered, which puts forward references to the maintenance distribution and resource checking of the constructing RTSN.
\end{abstract}

Keywords: The Railway Time Synchronization Network, Bayesian Networks, Common Cause Failure, Reliability Evaluation

\section{Introduction}

With the high speed railway speed increasing and driving distance shortening in China, each time synchronization of subsystems directly influences the punctuality of the giving and execution of the train order. And the RTSN is becoming more and more significant for it is the basic support network of railway communication, information and other networks [1-2].

The current railway clock synchronization network has been functioning for ten years. Most of the equipment is beyond their life span and the node coverage area is not enough. In 2008, China Railway Cooperation decided to establish new RTSN. Now, the 1 st and 2nd node have been completed. The 3rd one is under construction along with the construction of high-speed railway and dedicated passenger lines [3]. Therefore, analyses of reliability of the RTSN and the discovery of its weak links can provide references to the node checking and maintenance resource distribution of the constructing nodes [4].

In analyzing RTSN malfunction, much attention must be exerted to the influence from common cause failure (CCF), because time information in the time synchronization is synchronized from top to bottom according to tree structure. The upper unit malfunction impacts equipment of the same level and ones of lower level as well. If CCF is not taken into account in analyzing RTSN malfunction, the calculated failure rate of RTSN will be lower than that of the fact [5-6]. 
$\mathrm{BN}$ is known for its structure learning, updating learning and inference functions. Thus with BN, the reliability analysis model established by sample learning is more objective. Updating learning is suitable in reliability analyses of equipment with unknown malfunction model and data, for it can update and add malfunction model after the model is established [7]. Compared with current reliability analyses methods like fault tree analysis [8-10], petri network method [11-14], BN analysis has huge advantage in solving problems of establishing RTSN. The paper uses BN analysis, and with establishing the model by sample learning, analyzes the reliability of RTSN.

\section{Introduction to RTSN}

\subsection{Railway Time Synchronization}

Time synchronization means the calibration of time information connected with synchronization equipment with UTC, coordinating the former with the later. The railway time synchronization is that all railway bureaus and equipment in all stations must coordinate their time with China Railway Corporation in order to make sure the execution time of orders received matches the train working diagram from the headquarter[15].

\subsection{Introduction to RTSN}

There are three layers in RTSN and each of them provides time reference to different equipment. As is shown in Figure. 1, the first layer sets in the dispatch control center in headquarters, and time information is directly received from satellites by redundancy of GPS receiver and Beidou System (BDS) receiver. The second layer is distributed in every railway bureau, receiving time information from the clock of the first layer. When time information receiving fails, redundancy measures will be implemented, that is, time information is received from satellites by all the GPS receivers in each railway bureau. The third layers set in each station, section and receive time information from clocks of the second level, and when the time information cannot be received, redundancy measures will be applied, which means time information from near stations will be received. In the synchronization, time reference information is passed from the first layer to the second layer and then from the second to the third through the tree structure. Time synchronization equipment is only allowed to receive time information from higher level layer but not the same level nor the information from lower level to higher one[16]. As shown in Figure.1, the first layer mainly synchronizes DMS system clock; the second layer in each railway bureaus mainly synchronizes RBC,TSRS and signal focus detection system clocks; the third layer in every station synchronizes the computer interlocking system, TCC clocks[17].The synchronization uses NTP (Network Time Protocol) and RS422 (Application-layer Protocol) [18]. 


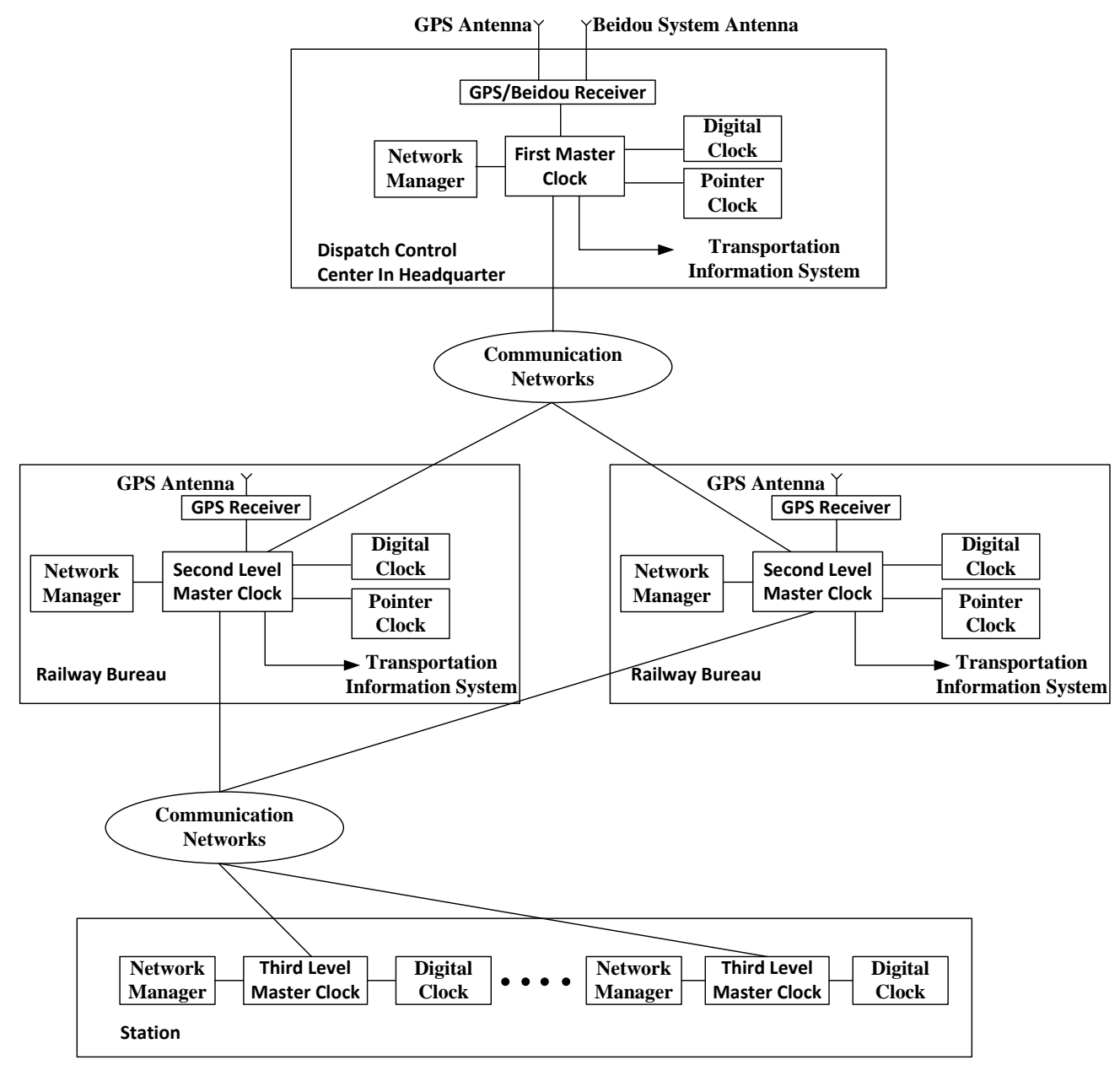

Figure 1. Railway Time Synchronization Network Framework

\section{Byesian Network}

BN network is a new way in AI area to process uncertainty problems. It is a graphical network based on probabilistic and statistics theory and can be applied to problems of uncertainty and incompleteness, especially ones like uncertainty malfunction of subsystems of large complex network, CCF and multiple failure modes. Because BN network has learning and reasoning ability. Currently it is widely used in the area of reliability analysis [19]. The reliability analysis process is in Figure. 2.

\subsection{Byesian Network Formula}

Up to now, probability theory is the most reliable reliability analysis method. Bayesian's theorem, describing the relationship of $\mathrm{N}$ conditional probabilities, was put forward by English scholar Thomas Bayesian based on the conditional probability calculation method in probability theory. Providing $A_{1}, A_{2}, \cdots A_{n}$ is not being consistent complete event group of $\mathrm{E}$ and $p\left(A_{i}\right)>0$ and $\mathrm{B}$ is the arbitrary event of $\mathrm{E}$, then the Bayesian's theorem as in (1). 


$$
p\left(A_{i} \mid B\right)=\frac{p\left(A_{i}\right) p\left(B \mid A_{i}\right)}{\sum_{j=1}^{n} p\left(A_{i}\right) p\left(B \mid A_{i}\right)}
$$

The $P(A i \mid B)$ is the probability that $\mathrm{Ai}$ happens under the condition that $\mathrm{B}$ happens; $P\left(A_{i}\right)$ is $\mathrm{A}_{\mathrm{i}}$ 's prior probability and $P(A \mid B)$ is the posteriori probability of $\mathrm{A}$. The discrete random variable form of Bayesian's theorem as in (2).

$$
p\left(X_{i}=x_{i} \mid Y=y\right)=\frac{p\left(X_{i}=x_{i}\right) p\left(Y=y \mid X_{i}=x_{i}\right)}{\sum_{j=1}^{n} p\left(X_{j}=x_{j}\right) p\left(Y=y \mid X_{j}=x_{j}\right)}
$$

If the probability obeys continuous random variable and assuming the probability of joint distribution of random variable $\xi, \eta$ is $p(x, y)=p_{\varepsilon}(x) f_{\eta \mid \varepsilon}(y \mid x)$ in which $p_{\varepsilon}(x)$ is the edge density of $\xi$ and $f_{\eta \mid \varepsilon}(y \mid x)$ is the conditional density of $\eta$ to $\xi$ when $\xi=\mathrm{x}$, thus the conditional density, $\mathrm{g}_{\xi \mid \eta}(\mathrm{x} \mid \mathrm{y})$ of $\xi$ to $\eta$ as in (3).

$$
g_{\varepsilon \mid \eta}(x \mid y)=\frac{p(x) f_{\eta \mid \varepsilon}(y \mid x)}{\int p_{\varepsilon}(x) f_{\eta \mid \varepsilon}(y \mid x) d x}
$$

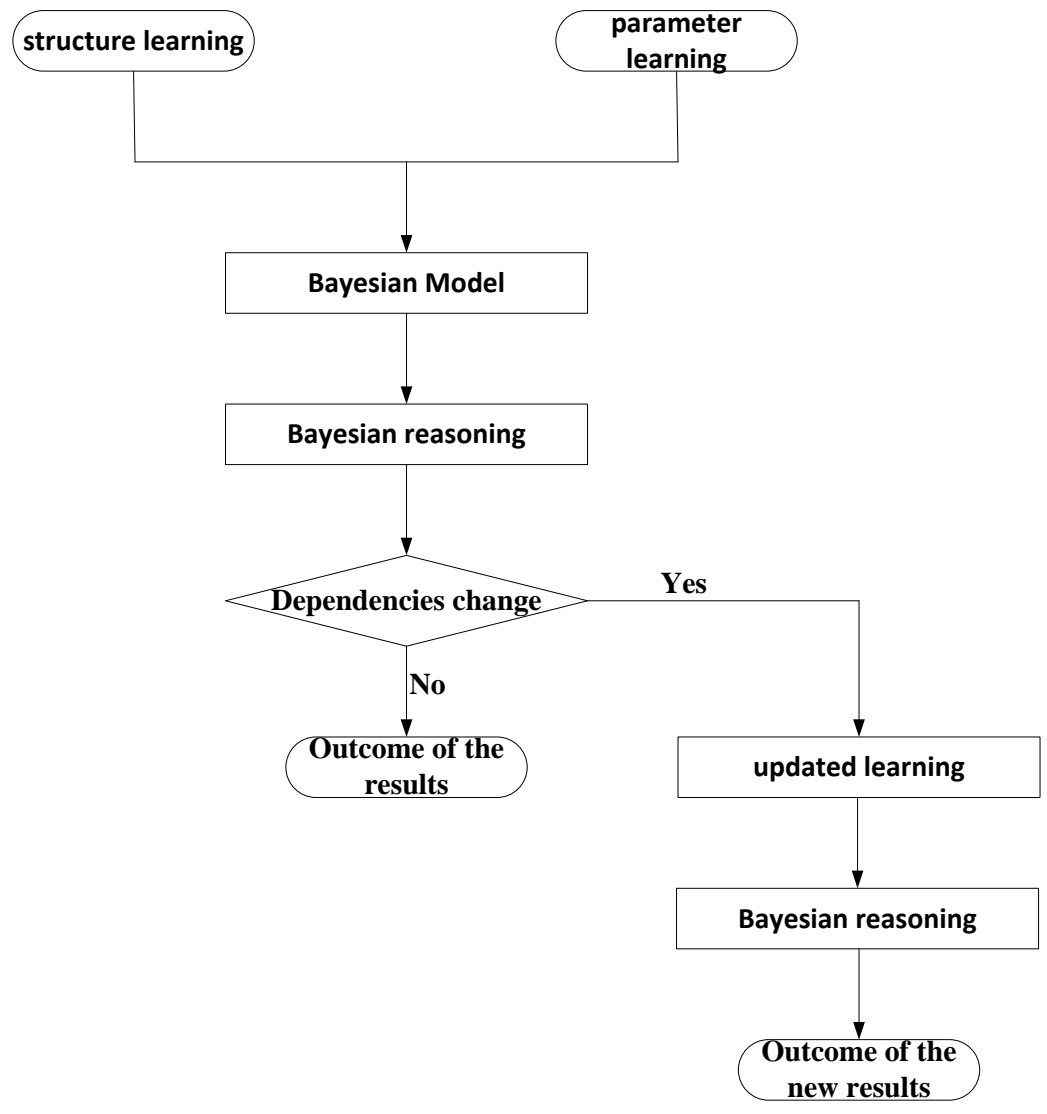

Figure 2. Reliability Analysis Process 


\subsection{Bayesian Network}

BN network approach can be divided into Bayesian learning and Bayesian reasoning. Bayesian learning has two parts---structure learning and parameter learning. By the learning of field data and expert sample, structure with the most posteriori probability of root node can be yielded from large numbers of produced structure combinations and it is the most accurate model in conducting reliability analysis. After the establishment of BN model, causal reasoning, diagnosis reasoning and supporting reasoning are applied to it in order to analyze the reliability index and weak links [20].

3.2.1. Advantage of Structure Learning: The advantage of $\mathrm{BN}$ network in analyzing problems lies in:(1) BN network is capable of structure learning and can produce the optimal network structure through massive field data and expert suggestions. Compared with fault tree analysis, cloud model analysis and petri net analysis, this method avoids subjectivity caused by manual operation in establishing models. The network produced from samples is more objective. (2) What differentiates BN network and the other reliability analyses is that BN network has updated learning ability. BN network is the model of dependency relationship among variables, and this dependency relationship may change with the change of environment. New dependency relationship will be also discovered with deeping understanding of the scene. The RTSN is under construction, so it is possible that field data and expert suggestions are not complete. When new malfunction model and dependency relationship are discovered, the $\mathrm{BN}$ model can add these by updated learning. (3) When expert suggestions are not complete, other massive samples can mend it. Thus it allows the expert to give the blurred advice when they come the uncertain problems, as shown in Table 1. This is useful in analyzing problems like CCF and multiple failure model.

Table 1. Expert Suggestions

\begin{tabular}{|c|c|l|l|}
\hline $\begin{array}{c}\text { Confirm } \\
\text { malfunction cause }\end{array}$ & $\begin{array}{c}\text { Possible } \\
\text { cause }\end{array}$ & $\begin{array}{l}\text { Confirm malfunction } \\
\text { model }\end{array}$ & Possible model \\
\hline $\begin{array}{c}\text { First master } \\
\text { clock malfunction }\end{array}$ & - & $\begin{array}{l}\text { This railway bureau has } \\
\text { not received time }\end{array}$ & $\begin{array}{l}\text { Near bureaus have not } \\
\text { received time information } \\
\text { information from China Railway } \\
\text { feadquarter. Synchronization } \\
\text { failed. }\end{array}$ \\
\hline
\end{tabular}

Table 2 shows the method of arranging sample data.

Table 2. Sample Data

\begin{tabular}{|l|l|l|l|}
\hline Malfunction cause & \multicolumn{3}{|c|}{ Malfunction model } \\
\hline $\mathrm{A}_{2}$ & $\mathrm{~B}_{1}$ & $\mathrm{~F}_{1}$ & $\mathrm{~B}_{2}$ \\
\hline 1 & 1 & 1 & $\mathrm{X}$ \\
\hline
\end{tabular}

In the table 2 above, 1 represent the event has happened and 0 represent the event has not happened. X means it is uncertain to recognize if the event has happened. When there are large numbers of samples, BN network can use other samples to repair some samples' lost data in its learning process, which makes it easier for experts to provide suggestions and makes it more objective for experts in analyzing uncertain problems. 
3.2.2. Bayesian Reasoning: There are three kinds of reasoning in $B N$ network: causal reasoning, diagnosis reasoning and supporting reasoning. Causal reasoning is used for get failure of the system. Diagnosis reasoning and supporting reasoning are used for seeking weak links in systems.

\section{Common Cause Failure}

Common cause failure (CCF) is a kind of failure which caused by the same reason and lead to two or more equipments in one system fail at the same time.

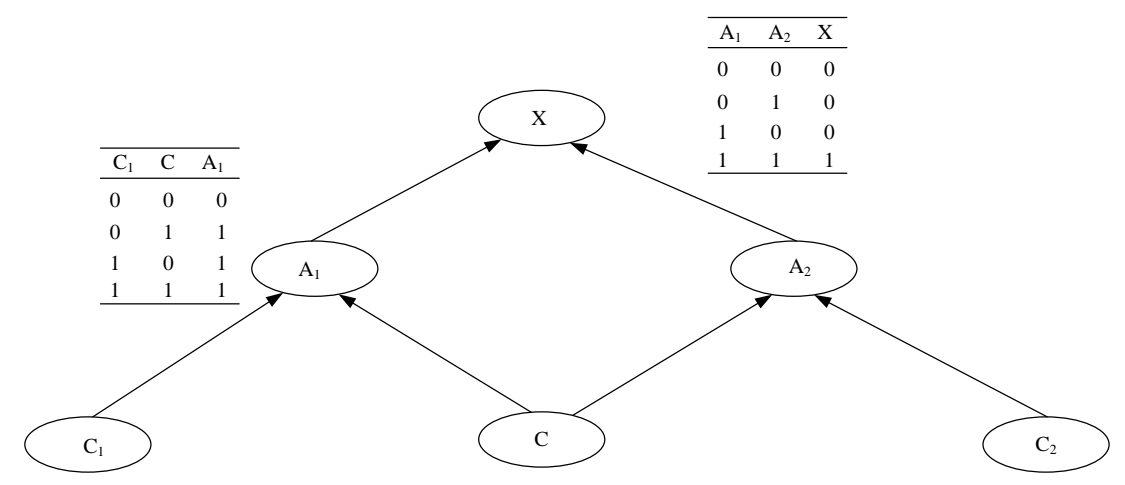

Figure 3. The CCF Model of BN

Figure 3 shows the CCF model of Bayesian. A1, A2 is the redundant equipment of system $\mathrm{X}$. and has two states " 1 " and " 0 ". 1 present the equipment is failure and 0 present the equipment is normal operation. $\mathrm{C} 1$ and $\mathrm{C} 2$ is the independent failure factor of $\mathrm{A} 1, \mathrm{~A} 2$. C is the common cause failure factor of A1, A2. The failure of whole system as in (4).

$$
\lambda_{\mathrm{CCF}}=\lambda_{\mathrm{C} 1} \lambda_{\mathrm{C} 2}+\lambda_{\mathrm{C}}
$$

Figure 4 shows the redundant optical's reliability in the case of considering and unconsidering CCF. It can be seen that the reliability which unconsidered CCF is significantly higher than the reliability which considered CCF. So the CCF is the main root to redundancy system and can't be ignored in the analysis of reliability issues.

The common cause failure rate is the difficult problem in analyzing CCF as it is hard to measure. The $\beta$ model, a model and MGL model are always used for analyzing CCF. The paper adopts $\beta$ model to analyze the CCF. As show in Figure 5, The value of $\beta$ would make a big influence on the result of analysis. According to majority of experiments in the real, the $\beta$ of hardware sets $10 \%$. 


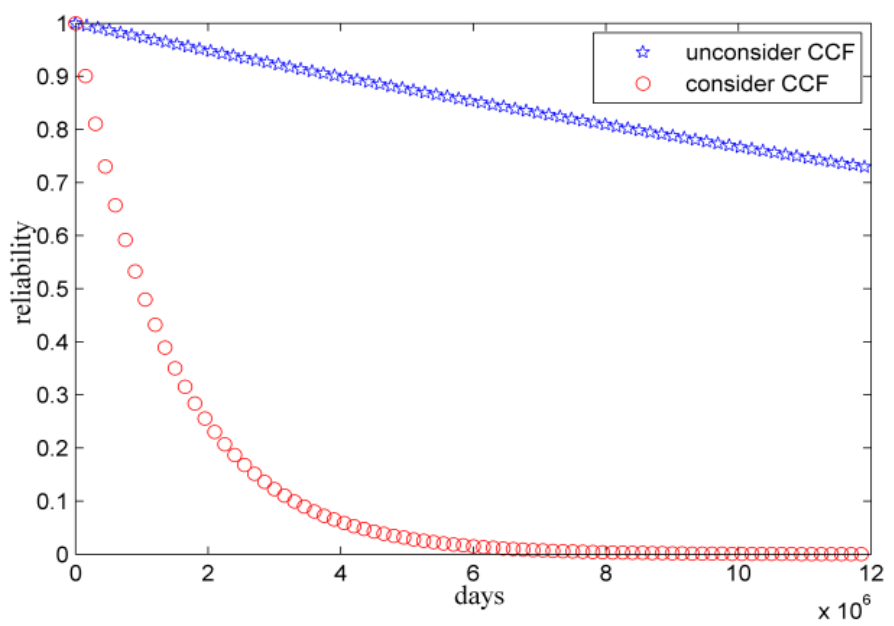

Figure 4. Reliability of CCF

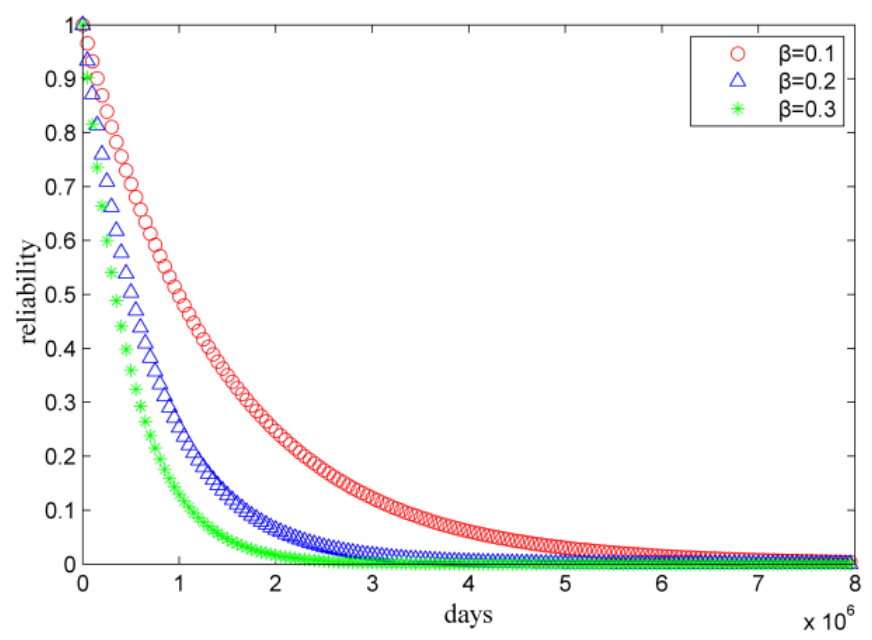

Figure 5. Reliability of Different B

The paper get the failure rate of all equipments according to the factory parameters of equipments and the data from scene. AS shown in table 3.

Table 3. The Failure of Equipments in RTSN

\begin{tabular}{lll}
\hline $\begin{array}{l}\text { Event } \\
\text { Code }\end{array}$ & Event Name & $\begin{array}{l}\text { Failure } \\
\text { Rate }(/ \mathrm{h})\end{array}$ \\
\hline $\mathrm{A}_{1}$ & GPS receiver malfunction & $5.1427 \times 10^{-7}$ \\
$\mathrm{~A}_{2}$ & Beidou receiver malfunction & $4.2148 \times 10^{-7}$ \\
$\mathrm{~A}_{3}$ & First level master clock malfunction & $1.0589 \times 10^{-8}$ \\
$\mathrm{~A}_{4}$ & SCADA interface sever malfunction & $3.2321 \times 10^{-6}$ \\
$\mathrm{~A}_{5}$ & DMS clock server malfunction & $3.7824 \times 10^{-6}$ \\
$\mathrm{~A}_{6}$ & Railway bureau GPS receiver malfunction & $5.1427 \times 10^{-7}$ \\
\hline
\end{tabular}




\begin{tabular}{lll}
\hline $\mathrm{A}_{7}$ & Optical fiber form CRC to railway bureaus malfunction & $2.1405 \times 10^{-4}$ \\
$\mathrm{~A}_{8}$ & Railway bureau second level master clock malfunction & $1.0589 \times 10^{-8}$ \\
$\mathrm{~A}_{9}$ & RBC interface sever malfunction & $6.3589 \times 10^{-6}$ \\
$\mathrm{~A}_{10}$ & TSRS sever malfunction & $5.3212 \times 10^{-6}$ \\
$\mathrm{~A}_{11}$ & CSM clock sever malfunction & $2.8252 \times 10^{-6}$ \\
$\mathrm{~A}_{12}$ & Autonomy machine malfunction & $3.8854 \times 10^{-6}$ \\
$\mathrm{~A}_{13}$ & TCC interface sever malfunction & $5.5262 \times 10^{-6}$ \\
$\mathrm{~A}_{14}$ & Optical fiber among bureaus malfunction & $6.7792 \times 10^{-6}$ \\
$\mathrm{~A}_{15}$ & Second level master clock malfunction (adjacent bureaus) & $1.0589 \times 10^{-8}$ \\
$\mathrm{~A}_{16}$ & Optical fiber among bureaus malfunction (adjacent & $6.7792 \times 10^{-6}$ \\
$\mathrm{~A}_{17}$ & bureaus) & $2.1405 \times 10^{-4}$ \\
$\mathrm{~A}_{18}$ & Optical fiber form CRC to railway bureaus malfunction & $5.1427 \times 10^{-7}$ \\
\hline
\end{tabular}

Table 3 shows the failure of equipments which unconsidered CCF. As railway time synchronization network is a safety critical system and majority of equipments in it contain redundant facility. So we must consider $\mathrm{CCF}$ in the analysis of reliability of railway time synchronization network. The Table 4 is established by synthesizing the data in Table 3 and sets $\beta$ is $10 \%$.

Table 4. The Failure of Equipments in RTSN Considered CCF

\begin{tabular}{lll}
\hline $\begin{array}{l}\text { Event } \\
\text { Code }\end{array}$ & Event Name & $\begin{array}{l}\text { Failure } \\
\text { Rate }(/ \mathrm{h})\end{array}$ \\
\hline $\mathrm{A}_{1}$ & GPS receiver malfunction & $5.14272 \times 10^{-8}$ \\
$\mathrm{~A}_{2}$ & Beidou receiver malfunction & $4.21481 \times 10^{-8}$ \\
$\mathrm{~A}_{3}$ & First level master clock malfunction & $1.0589 \times 10^{-9}$ \\
$\mathrm{~A}_{4}$ & SCADA interface sever malfunction & $3.23218 \times 10^{-7}$ \\
$\mathrm{~A}_{5}$ & DMS clock server malfunction & $3.78252 \times 10^{-7}$ \\
$\mathrm{~A}_{6}$ & Railway bureau GPS receiver malfunction & $5.14272 \times 10^{-8}$ \\
$\mathrm{~A}_{7}$ & Optical fiber form CRC to railway bureaus & $2.14421 \times 10^{-5}$ \\
$\mathrm{~A}_{8}$ & Ralfunction & $1.0589 \times 10^{-9}$ \\
$\mathrm{~A}_{9}$ & RBilway bureau second level master clock malfunction & $6.35923 \times 10^{-7}$ \\
$\mathrm{~A}_{10}$ & TSRS sever malfunction & $5.32143 \times 10^{-7}$ \\
$\mathrm{~A}_{11}$ & CSM clock sever malfunction & $2.82526 \times 10^{-7}$ \\
$\mathrm{~A}_{12}$ & Autonomy machine malfunction & $3.88552 \times 10^{-7}$ \\
$\mathrm{~A}_{13}$ & TCC interface sever malfunction & $5.52645 \times 10^{-7}$ \\
$\mathrm{~A}_{14}$ & Optical fiber among bureaus malfunction & $6.77957 \times 10^{-7}$ \\
\hline
\end{tabular}




\begin{tabular}{clc}
\hline $\mathrm{A}_{15}$ & $\begin{array}{c}\text { Second level master clock malfunction (adjacent } \\
\text { bureaus) } \\
\text { Optical fiber among bureaus malfunction (adjacent } \\
\mathrm{A}_{16}\end{array}$ & $1.0589 \times 10^{-9}$ \\
$\mathrm{~A}_{17}$ & $\begin{array}{c}\text { bureaus) } \\
\text { Optical fiber form CRC to railway bureaus } \\
\text { malfunction (adjacent bureaus) } \\
\text { Bureau GPS receiver malfunction } \\
\text { (adjacent bureaus) }\end{array}$ & $2.14421 \times 10^{-5}$ \\
$\mathrm{~A}_{18}$ & $5.14272 \times 10^{-8}$ \\
\hline
\end{tabular}

\section{Reliability Analysis of RTSN}

RTSN is a safety-critical system. The time reference information through it directly influences railway traffic safety, making it necessary to discover weak links in systems by reliability analysis.

This paper captures huge numbers of current time synchronization malfunction samples according to investigation of railway bureau and sample data obtained from experts' evaluation of relationship among each sub-element of RTSN. With learning of large numbers of sample data, the BN model of RTSN is established, as shown in Figure 6.

Conducting casual reasoning to $\mathrm{BN}$ network leads to the failure rate of RTSN: $\lambda(t)=3.09432 \times 10^{-6} / \mathrm{h}$, and the validity as in (4), the MTBF as in (5).

$$
\begin{aligned}
& A=1-\lambda(t)=0.9999690568 \\
& M T B F=1 / \lambda=323172.78 h
\end{aligned}
$$

Assuming $\mathrm{t}=10^{4} \mathrm{~h}$, the reliability of RTSN as in (6).

$$
R_{t}\left(2 \times 10^{4}\right)=e^{-\lambda t}=0.93999
$$

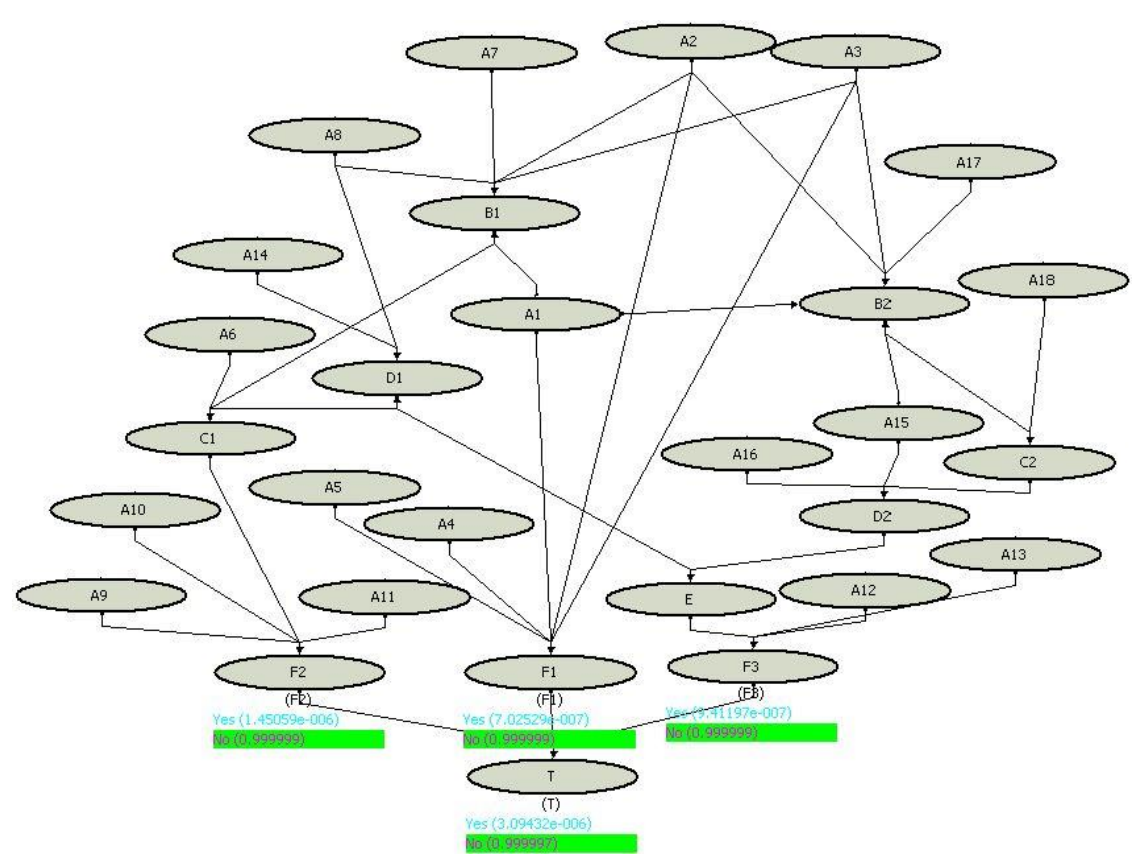

Figure 6. BN Model of RTSN 
Setting time as $2 \times 10^{5} \mathrm{~h}$, then the reliability is shown in Figure 7 .

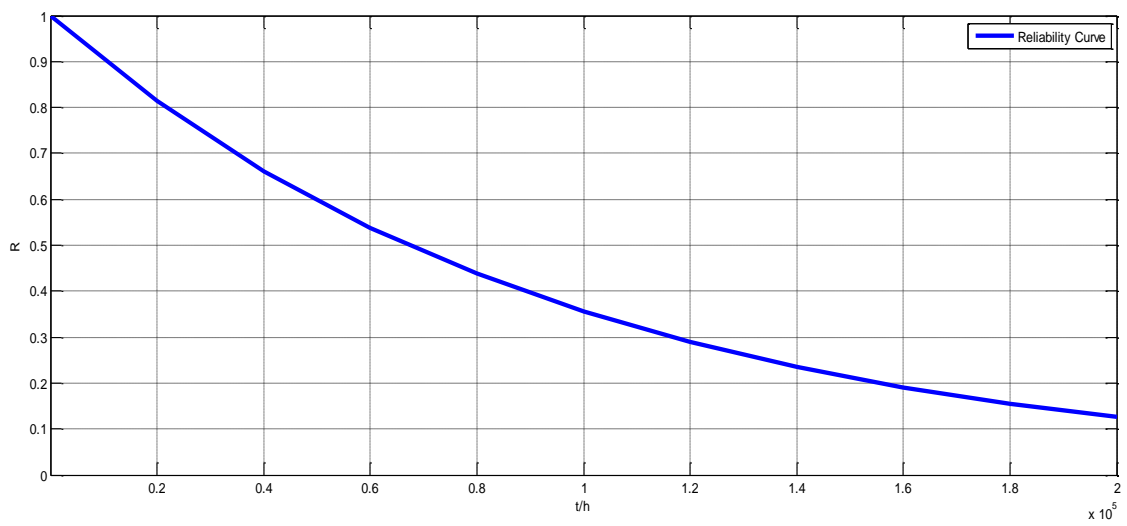

Figure 7. The Reliability Correlation Curve of Railway Time Synchronization Network

Table 5 is the standard of failure in railway. It is commanded that the failure of RSTN must satisfy the SIL 1 at least. The availability and MTBF must satisfy the requirement as shown in Table 6.

\section{Table 5. SIL Level}

\begin{tabular}{ll}
\hline SIL & Danger failure probability per hour \\
\hline SIL4 & $10^{-8} \leq \mathrm{THR}<10^{-7}$ \\
SIL3 & $10^{-7} \leq \mathrm{THR}<10^{-6}$ \\
SIL2 & $10^{-6} \leq \mathrm{THR}<10^{-5}$ \\
SIL1 & $10^{-5} \leq \mathrm{THR}<10^{-4}$ \\
\hline
\end{tabular}

According to Bayesian reasoning, the failure rate of RTSN is $3.09432 \times 10^{-6} / \mathrm{h}$ which meet the SIL1 level standard and the application requirements as shown in Table 5.

\section{Table 6. The RAM of RTSN}

\begin{tabular}{ll}
\hline & Standard \\
\hline Availability & $\geq 99.99 \%$ \\
MTBF & $\geq 3 \times 10^{5} \mathrm{~h}$ \\
\hline
\end{tabular}

The availability and MTBF must satisfy the requirement as shown in Table 6 . By counting the availability and MTBF, the availability is 0.9999690568 and the MTBF is $323172.78 \mathrm{~h}$ which satisfy the requirement.

Applying diagnosis reasoning to RTSN, if malfunction of RTSN happens, providing T=1 (RTSN malfunction), the diagnosis is shown in Table 7.

Table 7. Diagnosis Reasoning of RTSN

\begin{tabular}{llllll}
\hline Nodes & $\mathrm{A}_{1}$ & $\mathrm{~A}_{2}$ & $\mathrm{~A}_{3}$ & $\mathrm{~A}_{4}$ & $\mathrm{~A}_{5}$ \\
\hline Failure rate & 0.0348 & 0.0312 & 0.0003 & 0.0629 & 0.0699 \\
\hline
\end{tabular}




\begin{tabular}{llllll}
\hline Nodes & $\mathrm{A}_{6}$ & $\mathrm{~A}_{7}$ & $\mathrm{~A}_{8}$ & $\mathrm{~A}_{9}$ & $\mathrm{~A}_{10}$ \\
Failure rate & 0.0358 & 0.1128 & 0.0003 & 0.0758 & 0.0743 \\
Nodes & $\mathrm{A}_{11}$ & $\mathrm{~A}_{12}$ & $\mathrm{~A}_{13}$ & $\mathrm{~A}_{14}$ & $\mathrm{~A}_{15}$ \\
Failure rate & 0.0464 & 0.0742 & 0.0746 & 0.0794 & 0.0003 \\
Nodes & $\mathrm{A}_{16}$ & $\mathrm{~A}_{17}$ & $\mathrm{~A}_{18}$ & & \\
Failure rate & 0.0794 & 0.1128 & 0.0348 & & \\
\hline
\end{tabular}

According to Table VII, it is obvious that when the railway time synchronization network is failed, the most vulnerable parts of the fault is optical fiber form CRC to railway bureaus (A7 and A17) because its failure rate is the highest in all equipments. The weaker links are the optical fiber among bureaus and kinds of interface severs. From inference above, in order to increase the reliability of railway time synchronization network, we should pay much attention to maintenance of fiber, such as built the both direction circle redundancy network. We can use the way of 2 -vote- 3 or double 2 -vote- 2 to increase the reliability of interface severs.

Summarizing the above-mentioned, the paper gets three conclusions. (1) It is necessary to consider common cause failure in the analysis of redundant system, otherwise it will lead to the result which is significantly higher than reality. (2) The Bayesian network is good at solving common cause failure problem and seeking weak links of the system. It's proved that taking the $\mathrm{BN}$ into analysis of railway time synchronization network is feasible. (3) Fiber is the most vulnerable parts of railway time synchronization network. It's useful to pay much attention to maintenance of fiber in order to increase reliability of railway time synchronization network.

\section{Acknowledgements}

This project is supported by Railway Ministry Science and Technology Research and Development Program (2013X013-E).

\section{References}

[1] S. X. Wang, Editor, "Intelligent transportation railway system", China Railway Publishing House, Beijing, (2008).

[2] Q. Z. Wang, "Optimization scheme for the railway time synchronization network", Railway Signalling \& Communication, vol. 49, no.3, (2013), pp. 72-75.

[3] B. Qu, "Summarize of the railway time synchronization network", Railway Signalling \& Communication, vol. 7, no.4, (2010), pp. 42-44.

[4] Y. Su, "Design and implementation of time synchronization to railways information system", Railway Computer Application, vol. 13, no.1, (2004), pp. 20-23.

[5] D. Y. Shi, "The Research of railway time synchronization network", Railway Signalling \& Communication Engineering, vol. 9, no.1, (2012), pp. 38-40.

[6] L. Richard, G. Mohit, "A detailed study of NHPP software reliability models", Journal of Software, vol. 17, no.6, (2012), pp. 1296-1306.

[7] Y. C. Yao, N. D. Chen and B. Wang, "Fuzzy reliability assessment method based on T-S fault tree and Bayesian network", Journal of Mechanical Engineering, vol. 49, no.6, (2013), pp. 90-97.

[8] X. Feng, F. X. Wang, "Analysis on reliability and performance of computer-based interlocking System with the dynamic fault tree method", Journal of the China Railway Society, vol. 13, no.12, (2011), pp. 79-82.

[9] H. G. Li, Q. LI, G.J. Zhao and Q. Zhou, "Fault simulation analysis of mechanical firing based on fault tree", Journal of Machine Design, vol. 30, no.3, (2013), pp. 9-11.

[10] J. T. Cui, D. Y. Ma, "Research on multi-dimensional space fault tree construction and application", China Safety Science Journal, vol. 23, no.4, (2013), pp. 33-37.

[11] H. T. Xu, L. H. Zhao and T. Tang, "Colored-Petri-Nets based reliability analysis of ETCS train radio communication”, Journal of the China Railway Society, vol. 30, no.1, (2008), pp. 39-42. 
[12] J. Liu, L. Pan, "Blast furnace fault diagnosis system based on fuzzy neural Petri Net", Computer Measurement \& Control, vol. 21, no.5, (2013), pp. 1157-1162.

[13] Y. X. Tong, T. H. Xie and W. M. Sun, "Power system fault diagnosis model based on layered fuzzy petri net considering temporal constraint checking", Automation of Electric Power Systems, vol. 37, no.6, (2013), pp. 63-68.

[14] H. Yuan, "Principle \& application of railway time synchronization network", Telecommunications Technology, vol. 10, no.1, (2001), pp. 69-71.

[15] H. Chen, "The integration for the railway time synchronization network and the railway frequency synchronization network", Telecommunications Network Technology, vol. 9, no.9, (2012), pp. 51-53.

[16] Q. H. Lu, "The research for development of railway time synchronization network", Railway Signalling \& Communication Engineering, vol. 48, no.8, (2012), pp. 54-58.

[17] S. C. San, G. Steven, "A colored Petri net methodology and library for security analysis of network protocols", Journal of Computers, vol. 9, no.2, (2014), pp. 243-256.

[18] L. Wang, Q. Wang, "The discussion about the railway time synchronization network", Railway Transport and Economy, vol. 30, no.1, (2007), pp. 49-51.

[19] K. Q. Xiao, Editor, "The learning reasoning application of Bayesian”, National Defence Industry Press, Beijing, (2007).

[20] C. S. Wang, Editor, “The application of Bayesian”, Li xin Accounting Press, Shanghai, (2010).

[21] Q. B. Yu, W. Li and M. Zhang, "Redundancy transmission system based on multidisciplinary object compatibility design optimization”, Journal of Networks, vol. 9, no.3, (2014), pp. 754-761.

\section{Authors}

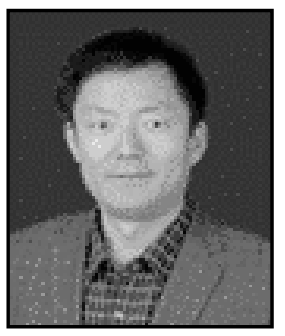

Youpeng Zhang, He is serving as a full-time professor and dean at school of Automation and Electrical Engineering, Lanzhou Jiaotong University. His research interest includes System Security and Reliability, Intelligent Control, Power Systems and Its Automation, and etc.

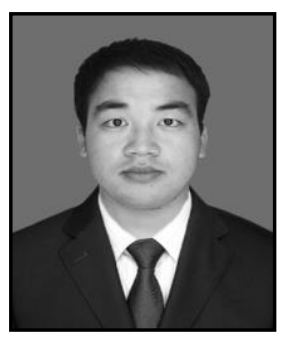

Feng Wang, He was born in Gansu, China, in 1989. He received his B. S. degree in Lanzhou Jiaotong University in 2010. Currently, he is a Master candidate in Traffic Information Engineering and Control in Lanzhou Jiaotong University, China. His recent research interest is reliability and safety analysis of Chinese railway time synchronization network.

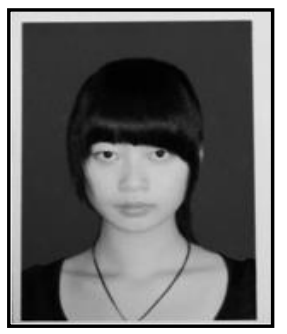

Shan Zhang, She was born in Anhui, China, in 1990. She received her B. S. degree in Lanzhou Jiaotong University in 2010. Currently, she is a Master candidate in Traffic Information Engineering and Control in Lanzhou Jiaotong University, China. Her recent research interest is the accuracy of Chinese railway time synchronization network. 


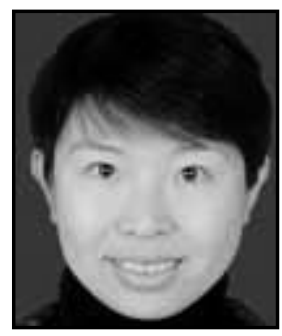

Li Lan, She is serving as a full-time lecturer at school of Electronics and Information Engineering, Lanzhou Jiaotong University. She is a $\mathrm{Ph}$. D. candidate in Lanzhou Jiaotong University. Her recent research interest is reliability and safety analysis of networks. 
International Journal of Control and Automation Vol.8, No.10 (2015) 